\title{
Effectiveness of a Layer-by-Layer Microbubbles-Based Delivery System for Applying Minoxidil to Enhance Hair Growth
}

\author{
Ai-Ho Liao ${ }^{1,2}$, Ying-Jui Lu1 ${ }^{1}$, Yi-Chun Lin ${ }^{3}$, Hang-Kang Chen ${ }^{3}$, Huey-Kang Sytwu ${ }^{3,4}$, Chih-Hung \\ Wang $3,4,5 \bowtie$ \\ 1. Graduate Institute of Biomedical Engineering, National Taiwan University of Science and Technology, Taipei 10607, Taiwan. \\ 2. Department of Medical Engineering, National Defense Medical Center, Taipei 11490, Taiwan. \\ 3. Graduate Institute of Medical Sciences, National Defense Medical Center, Taipei 11490, Taiwan. \\ 4. Graduate Institute of Microbiology and Immunology, National Defense Medical Center, Taipei 11490, Taiwan. \\ 5. Department of Otolaryngology-Head and Neck Surgery, Tri-Service General Hospital, National Defense Medical Center, Taipei 11490, Taiwan.
}

$\square$ Corresponding authors: Ai-Ho Liao, Ph.D. Graduate Institute of Biomedical Engineering, National Taiwan University of Science and Technology, TR-916, \#43, Sec. 4, Keelung Rd., Taipei 10607, Taiwan. Phone: +886-2-27303742 Fax: +886-2-27303742 E-mail: aiho@mail.ntust.edu.tw Or Chih-Hung Wang, M.D., Ph.D. Department of Otolaryngology-Head and Neck Surgery, Tri-Service General Hospital, National Defense Medical Center, 325, Sec. 2, Cheng-Kung Rd., Taipei 11490, Taiwan. Phone: +886-2-87927192 Fax: +886-2-87927193 E-mail: chw@ms3.hinet.net.

(C) Ivyspring International Publisher. Reproduction is permitted for personal, noncommercial use, provided that the article is in whole, unmodified, and properly cited. See http://ivyspring.com/terms for terms and conditions.

Received: 2016.01.11; Accepted: 2016.03.03; Published: 2016.04.11

\begin{abstract}
Minoxidil (Mx) is a conventional drug for treating androgenetic alopecia, preventing hair loss, and promoting hair growth. The solubility of $M x$ has been improved using chemical enhancement methods to increase its skin permeability over the long term. This study created a new ultrasound (US) contrast agent - albumin-shelled microbubbles (MBs) that absorb chitosan oligosaccharide lactate $(\mathrm{COL})$ and $\mathrm{Mx}$ - and combined it with sonication by US energy in the water phase to enhance hair growth while shortening the treatment period. COL and Mx grafted with MBs (mean diameter of $1480 \mathrm{~nm}$ ) were synthesized into self-assembled complexes of COL-MBs and Mx-COL-MBs that had mean diameters of 4150 and $4500 \mathrm{~nm}$, respectively. The US was applied at $3 \mathrm{~W} / \mathrm{cm}^{2}$ for $1 \mathrm{~min}$, and combined with $\mathrm{Mx}-\mathrm{COL}-\mathrm{MBs}$ containing $0.3 \% \mathrm{Mx}$. The diffusion of $\mathrm{Mx}$ through the dialysis membrane from Mx-COL-MB during US (US+Mx-COL-MB) was more rapid at $\mathrm{pH} 4$ than at $\mathrm{pH} 7.4$, which is favorable given that the environment of the scalp is mildly acidic ( $\mathrm{pH}=4.5-5.5)$. In Franz diffusion experiments performed in vitro, the release rates at 18 hours in the US+Mx-COL-MBs and US+MBs+Mx groups resulted in 2.3 and 1.7 times the penetration and deposition, respectively, of Mx relative to the group with $M x$ alone. During 21 days treatment in animal experiments, the growth rates at days 10 and 14 in the US+Mx-COL-MBs group increased by $22.6 \%$ and $64.7 \%$, respectively, and there were clear significant differences $(p<0.05)$ between the US+Mx-COL-MBs group and the other four groups. The use of US+Mx-COL-MB in the water phase can increased the effects of $M x$ so as to shorten the telogen phase, and also increase both the diameter of keratinized hair shafts and the size of hair follicles without causing skin damage.
\end{abstract}

Key words: Microbubbles, Ultrasound, Transdermal drug delivery, Minoxidil, Chitosan oligosaccharide lactate, Hair growth.

\section{Introduction}

Hair-loss disorders affect men and women of all ages, with the prevalence increasing with age [1, 2]. Androgenetic alopecia (AGA) is the most common form of hair loss, and it is characterized by specific patterns of temporal-frontal loss in men and central thinning in women [1]. AGA is induced by androgens in genetically susceptible hair follicles either in men or in women. In such hair follicles dihydrotestosterone 
(DHT) binds to the androgen receptor and this hormone-receptor complex then activates the genes that control the gradual transformation of large, terminal follicles into small, miniaturized follicles [3]. Topical minoxidil (Mx) (Rogaine) is the only medication that can be used by both men and women, and is approved by the US Food and Drug Administration (FDA) for the treatment of AGA [1]. However, it does not work on completely bald areas and only works over the long term if it is used continuously [1]. The present study created a new ultrasound (US) contrast agent-chitosan oligosaccharide lactate (COL) and Mx-coated microbubbles (MBs)-and combined it with sonication by US energy in the water phase [4] to enhance hair growth and reduce the treatment period.

Mx was introduced in the early 1970 s as a treatment for hypertension, and common side effects when taking $\mathrm{Mx}$ tablets include the regrowth of hair in male balding [5-7]. A $2 \%$ topical formulation was first marketed for hair regrowth in men in 1986 in the US, and the 5\% product was made available in 1993 [8]. The $2 \% \mathrm{Mx}$ solution was approved as a medication by US FDA for women, and both the $2 \%$ and 5\% solutions were made available for men [9]. Mx may stimulate hair growth by increasing the anagen phase of the hair cycle, but the exact mechanisms are still unclear [10]. In 2006, 5\% Mx was approved by the US FDA for treating AGA in men, and clinical testing showed that hair growth was increased after 48 weeks of using it twice daily $[10,11]$.

The relative concentrations of propylene glycol and ethanol in a binary solvent system had a significant effect on the skin penetration of $2 \% \mathrm{Mx}$ solution [12]. However, some patients present with clinical complaints of pruritus and scaling of the scalp [13], and it was found that this was mainly due to propylene glycol rather than to the Mx itself. Many alternative candidate treatment formulations using other solvents have been considered, such as butylene glycol, polysorbate, and glycerol. However, patch testing has indicated that the compounded preparations might not be tolerated [13]. Recently, chitosan microparticles loaded with $\mathrm{Mx}$ were prepared in an ethanol-water solution at a $\mathrm{pH}$ of 5.5 to reduce the immunogenicity of a topical alopecia therapy [14]. The follicular bioavailability of $\mathrm{Mx}$ from this formulation and the potential use of such microparticles in targeting drug delivery for the topical treatment of alopecia are still being evaluated [14]. Microneedling-induced hair growth in mice has been also recently reported [15, 16]. However, constructing suitable microneedles is problematic, and these needles may accidentally break and then remain in the skin [17].

Sonophoretic transdermal drug delivery has been used with MBs as a US contrast agent to enhance the delivery of drugs into the porcine skin via cavitation induced by high-frequency US [18, 19]. More recently, it was reported that combined treatment with optimal US parameters $\left(3-\mathrm{W} / \mathrm{cm}^{2}\right.$ for 1 $\min )$ and MBs $\left(2.9 \times 10^{8} / \mathrm{ml}\right)$ can increase the skin permeability so as to enhance the delivery of a-arbutin to inhibit melanogenesis without damaging the skin in mice [20]. Combining US with MBs of different sizes can produce different degrees of skin permeability, thereby making it possible to enhance the delivery of even large molecules (>500 Da) [21].

The present study created a new type of Mx-coated MBs and combined them with sonication by US energy in the water phase to enhance hair growth while shortening the treatment period. COL was grafted as the first layer on albumin-shelled MBs based on the protective effect of COL for human dermal fibroblast cells [22]. The complex was mixed with $\mathrm{Mx}$ solution to prepare self-assembled Mx-COL-MBs (Fig. 1). The use of US-mediated Mx-COL-MBs cavitation to induce Mx delivery and enhance hair growth in vivo was also investigated.

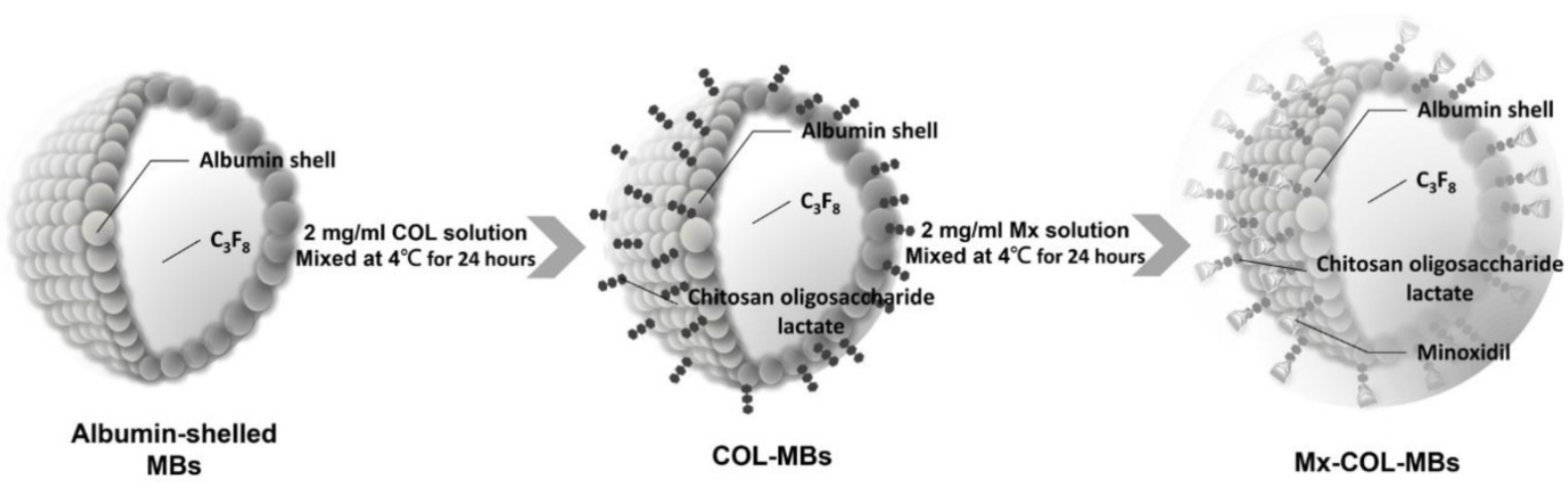

Figure 1: Schematic (not to scale) of the layer-by-layer self-assembly of Mx-COL-MBs. 


\section{Materials and Methods}

\section{Preparation characterization of Mx-COL-MBs}

Self-assembled Mx-COL-MBs (Fig. 1) were prepared, whose composition is presented in Fig. 1 and Table 1. Since albumin has negative charges, the surface potential of the albumin shell is less than zero, and thus, it can attract the molecule with positive charges. In an acidic solution, COL has a plurality of amine $\left(-\mathrm{NH}_{2}\right)$ which absorbs hydrogen ions, and changes into $-\mathrm{NH}_{3}{ }^{+}$(positive charge). Therefore, the albumin shell with negative charges can be adsorbed onto COL by electrical adsorption. This makes the COL distributed on the outside shell surface of albumin, and becomes the COL-MB through the modification by COL. Mx has the negatively charged oxygen atoms, which is easily attracted by the $-\mathrm{NH}_{3}{ }^{+}$ of COL, and becomes the Mx-COL-MB. Albumin-shelled MBs were prepared according to the procedure used in our previous study [23, 24]. HSA was purchased as a sterile $20 \%$ solution (Octapharma, Vienna, Austria), and it was diluted with PBS to make stock solutions containing $1.32 \%(w / v)$ HSA. MBs were generated by sonication in $10 \mathrm{ml}$ of solution in perfluorocarbon gas in PBS using a sonicator $(200 \mathrm{~W}$; Branson Ultrasonics, Danbury, CT, USA) for 2 min. The MBs were centrifuged at $1200 \mathrm{rpm}(128.6 \times g)$ for 1 minute, and the subnatant was removed. A 1-ml aliquot of $2 \mathrm{mg} / \mathrm{ml} \mathrm{COL} \mathrm{(molecular} \mathrm{weight=5000,}$ Sigma-Aldrich, St. Louis, MO, USA) solution was then mixed and stirred (50 rpm) for 24 hours at $4^{\circ} \mathrm{C}$. The COL-MBs were centrifuged at $1200 \mathrm{rpm}(128.6 \times \mathrm{g})$ for 1 minute, and then washed three times to eliminate the free COL. The $2 \mathrm{mg} / \mathrm{ml} \mathrm{Mx}$ (molecular weight $=209.25$, Sigma-Aldrich) solution was then mixed in various volume ratios of COL $(1: 1,1: 2$, and $1: 3)$ and stirred $(50 \mathrm{rpm})$ for 24 hours at $4^{\circ} \mathrm{C}$. The Mx-COL-MBs were centrifuged at $1200 \mathrm{rpm}(128.6 \times \mathrm{g})$ for 1 minute, and then washed three times to eliminate the free Mx. The number of Mx-COL-MBs in the solution was measured with the MultiSizer III device (Beckman Coulter, Fullerton, CA) using a $30-\mu \mathrm{m}$ aperture probe whose measurement boundary ranged from 0.6 to $20 \mu \mathrm{m}$. The zeta potentials of the MBs, COL-MBs, and Mx-COL-MBs dispersed in an aqueous solution $(\mathrm{pH}=6.4$, resistance $=18.2 \mathrm{~m} \Omega$ ) were measured using a Nanoparticle Analyzer (Horiba, Kyoto, Japan). The albumin-shelled MBs, COL-MBs, and Mx-COL-MBs were filtered with a $5-\mu \mathrm{m}$ syringe filter (Sartorius, Goettingen, Germany) and then hardened using $0.25 \%$ glutaraldehyde (SigmaAldrich). The morphology of the hardened MBs was studied using transmission SEM after coating the samples with platinum (achieved by applying $20 \mathrm{~mA}$ for $20 \mathrm{~min}$ ). SEM images were recorded on an automatic sputter coater (JFC-1300, JEOL, Tokyo, Japan) at an accelerating voltage of $15 \mathrm{kV}$.

\section{In vitro release study}

The in vitro release behaviors of $\mathrm{Mx}$ from $\mathrm{MB}$ vesicles were investigated using a dialysis method. Figure 2 shows a schematic of the system setup. Briefly, $3 \mathrm{ml}$ of an Mx-COL-MBs suspension (of the original concentration after production) was loaded into a dialysis bag (molecular weight cutoff $=12-14$ $\mathrm{kDa}$ ) and dialyzed against the release media of PBS at $\mathrm{pH}$ values of 4.0 and 7.4 within $0.5^{\circ} \mathrm{C}$ of $37.0^{\circ} \mathrm{C}$, and with stirring by a magnetic bar at $600 \mathrm{rpm}$. After 0.5 hours, the 1-MHz unfocused US therapy transducer of a sonoporation system (ST 2000V, NepaGene, Ichikawa, Japan) positioned $3 \mathrm{~mm}$ from the top of the dialysis bag under the liquid level provided sonication at a power density of $3 \mathrm{~W} / \mathrm{cm}^{2}$ (acoustic pressure $=0.266 \mathrm{MPa}$ ) for $1 \mathrm{~min}$. One-milliliter samples were taken from the release medium after times of 0.1 , $0.2,0.3,0.4,0.5,1,2,3,4,5$, and 6 hours, and the same volume of PBS was added to replace it in the release medium. These samples were kept in a freezer until analyzed using a UV-visual spectrophotometer (Lambda 40, Perkin Elmer, Bridgeville, PA, USA). The mean values obtained from four replicates were calculated. The drug release profile of $\mathrm{Mx}$ was examined as a control. The accumulative release percentage of $\mathrm{Mx}$ from $\mathrm{MB}$ was calculated according to the following equation [25]:

$$
R=\frac{c_{n} v_{0}+\sum_{i=0}^{n-1} c_{i} v_{i}}{W} \times 100 \%
$$

where $R$ is the release rate, $c_{n}$ is the drug concentration in the release medium at each time point, $v_{0}$ is the total volume of the release medium $(100 \mathrm{ml}), v_{i}$ is the volume of the withdrawn medium every time $(1 \mathrm{ml})$, $c_{i}$ is the drug concentration in the release medium at intervals of $i$, and $W$ is the mass of drug used for release [25].

Table 1. Composition, zeta potential, diameter, and Mx encapsulation efficiency of various MBs.

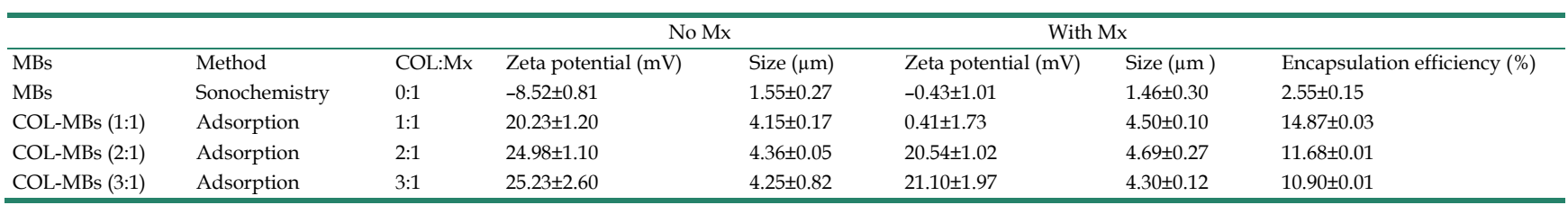



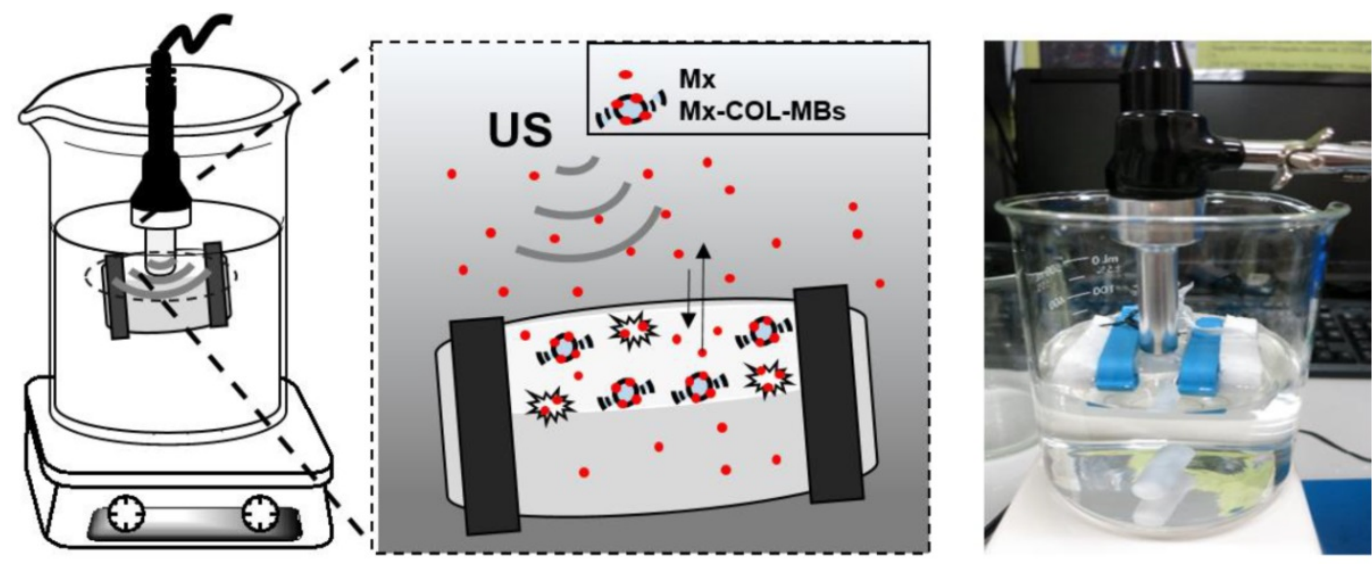

Figure 2: Schematic diagram of in vitro release rate behaviors of $M x$ from $M B$ vesicles.

\section{Measurements of penetration depth in pigskin}

Fresh porcine ear skin with hair follicles was obtained from the Affiliated Slaughterhouse of New Taipei City Meat Market, and all experiments on them were completed within 6 hours. Circular porcine ear skin samples were constructed with a radius of $1.2 \mathrm{~cm}$ and a thickness of $3 \mathrm{~mm}$ (encircled with US gel to prevent leakage); the round area of each pigskin sample was loaded with FITC or MBs. The treatment area of the sample was sonicated by the 1-MHz US transducer of the sonoporation system attached to the top of the sample under the liquid level, and was performed successively at a power density of $3 \mathrm{~W} / \mathrm{cm}^{2}$ (acoustic pressure $=0.266 \mathrm{MPa}$ ) for $1 \mathrm{~min}$ after adding $500 \mu \mathrm{l}$ of the MBs with $0.1 \mathrm{mg}$ of FITC. The MBs and FITC solution was left for 6 hours after sonication, removed, and then the area was washed three times for $1 \mathrm{~min}$ each with PBS. The treated areas of pigskin were removed and then embedded in an optimal-cutting-temperature solution (Surgipath FSC 22, Leica Microsystems, Buffalo Grove, IL, USA) on round specimen disks with a diameter of $2.2 \mathrm{~cm}$. The embedded samples were placed on the $-25^{\circ} \mathrm{C}$ freezing stage of a cryostat (Microm HM550 series, Thermo, Braunschweig, Germany) for about $30 \mathrm{~min}$. Transverse sectioning was performed at a slice thickness of $10 \mu \mathrm{m}$. Sections attached to the microscopy slides were air-dried at room temperature and mounted for microscopy examination. The distribution of the FITC in the cryosections was determined by Upright fluorescence microscope in the transmission and fluorescence modes (DM 2500, Leica Microsystems, Wetzlar, Germany) [26].

\section{In vitro skin penetration by $\mathbf{M x}$}

Fresh porcine ear skin with hair follicles was obtained from the Affiliated Slaughterhouse of New Taipei City Meat Market, and all experiments on them were completed within 6 hours. A 2-mm-thick sample of pigskin was harvested using a Humby knife, carefully cleaned with PBS, and cut into square pieces $(2 \mathrm{~cm} \times 2 \mathrm{~cm})$. The in vitro skin penetration was then tested using static Franz diffusion cells (Fig. 3) over an area of $2.14 \mathrm{~cm}^{2}$ according to the experimental design used in our previous study [20]. The temperature of the diffusion assembly was maintained at $37^{\circ} \mathrm{C}$. The probe of the sonoporation system, MBs, $0.3 \mathrm{mg}$ of $\mathrm{Mx}$ (molecular weight=209.25, Sigma-Aldrich), or Mx-COL-MBs (containing $0.3 \mathrm{mg}$ of $\mathrm{Mx}$ ) in PBS (1 ml) (as a control) were applied to the donor cells facing the stratum corneum side of the skin, and occluded with Parafilm (Pechiney Laboratory Safety Products and Apparel, Chicago, IL, USA). The receptor diffusion half-cell facing the dermis side was filled with PBS ( $\mathrm{pH} 7.4,12 \mathrm{ml}$ ); that cell contained a magnetic stirring bar rotating at $600 \mathrm{rpm}$ and $0.01 \%$ gentamicin to prevent bacterial degradation of the $\mathrm{Mx}$ during the penetration process. Solutions in the diffusion cell without MBs were filtered through a $0.2-\mu \mathrm{m}$ micropore filter (Nalgene, Rochester, NY, USA) or a $0.22-\mu \mathrm{m}$ micropore filter (Millex, Darmstadt, Germany). Aliquots $(200 \mu \mathrm{l})$ of receptor solution were taken after various time points $(0.5,1,2$, $3,4,5,6,8,12$, and 18 hours), with the cell refilled each time with the same volume of fresh receptor solution. After 0.5 hours, the $1-\mathrm{MHz}$ US transducer of the sonoporation system (ST 2000V, NepaGene) positioned $3 \mathrm{~mm}$ from the top of the skin provided sonication at a power density of $3 \mathrm{~W} / \mathrm{cm}^{2}$ (acoustic pressure $=0.266 \mathrm{MPa}$ ) for $1 \mathrm{~min}$. Samples were kept in a freezer until analyzed by a UV-visual spectrophotometer (Lambda 40, Perkin Elmer).

At the end of the penetration experiments (i.e., after 18 hours), the skin sample was detached from the diffusion cell and carefully rinsed five times with distilled water to remove excess $\mathrm{Mx}$ from its surface. The skin was cut into 0.1-g pieces and homogenized with $1 \mathrm{ml}$ of receptor solution for $2 \mathrm{~min}$ at 10,000 rpm (Polytron-Aggregate PT3100, Kinematica, Luzern, 
Switzerland). The homogenized suspension was centrifuged for $25 \mathrm{~min}$ at 3,100×g (Thermo Fisher Scientific, Bremen, Germany) and then the concentrations of $\mathrm{Mx}$ in the supernatant were determined using a UV-visual spectrophotometer. Sample volumes of $200 \mu \mathrm{l}$ were added to the cuvette and placed in the spectrophotometer. The $\mathrm{Mx}$ calibration curve served as the standard curve against which the absorption peaks and the corresponding concentrations of $\mathrm{Mx}$ in the samples were measured.

\section{Animal treatments}

Six-week-old C57BL/6 mice weighing 20-25 g were obtained from Bio Lasco (Taipei, Taiwan). The experimental protocol was approved by the Institutional Animal Care and Use Committee of the National Defense Medical Center, Taipei, Taiwan. Animals were cared for in compliance with institutional guidelines and regulations. Throughout the experiments, the animals were housed in stainless-steel cages in an air-conditioned room with the temperature maintained at $25-28^{\circ} \mathrm{C}$ and with alternating light and dark periods of 12 hours each. The animals were acclimatized for 7 days prior to the experiments. An area of about $10 \mathrm{~cm}^{2}$ on the dorsal skin of each animal was shaved using an animal clipper when the animal was 8 weeks of age, at which time all of the hair follicles were synchronized in the telogen phase, and the skin color was measured using the CR-400 Chroma Meter device (Konica Minolta Sensing, Tokyo, Japan). The animals were divided into the following five groups $(n=6$ per group, treatment applied once daily for 3 weeks): (i) no treatment (C group), (ii) penetrating $\mathrm{Mx}$ alone ( $\mathrm{Mx}$ group), (iii) US combined with penetrating Mx (US group), (iv) US combined with MBs and penetrating Mx (US+MBs group), and (v) US combined with Mx-COL-MBs (US+Mx-COL-MBs group). The US was applied at $3 \mathrm{~W} / \mathrm{cm}^{2}$ (acoustic pressure $=0.266 \mathrm{MPa}$ ) for $1 \mathrm{~min}$, and $0.3 \mathrm{mg} / \mathrm{ml}\left(0.5 \mathrm{ml} / \mathrm{cm}^{2}\right) \mathrm{Mx}$ was used in all cases. The change in skin color induced by each of the treatments was assessed at predetermined times using the Chroma Meter. The luminosity index, L [27], was calculated on each measurement day before and after treatment. The hair growth rate was calculated according to the $L$ value using the following equation:

$$
\text { Hair growth rate }(\%)=\frac{L_{1}-L_{n}}{L_{1}} \times 100 \%
$$

where $L_{1}$ is the luminosity index immediately after removing the hair and $L_{n}$ is the luminosity index at each measurement time point.

\section{Histochemistry}

Skin tissue samples (approximately $8 \mathrm{~mm} \times 8$ $\mathrm{mm}$ ) were cut from the treatment area immediately after the experiments and stored in a 10\% formalin solution. Hematoxylin and eosin (Sigma-Aldrich) staining was applied, and the thickness, diameter, and number of hair follicles were analyzed by an image analysis system (TissueFAXS 3.5, TissueGnostics, Vienna, Austria) using both the scanner (TissueQuest) and cytometry (HistoQuest) analysis packages provided with the system.

\section{Statistical analysis}

The obtained data were analyzed statistically using Student's $t$-test. A probability value of $p<0.05$ was considered indicative of a significant difference. Data are presented as mean $\pm S D$ values.

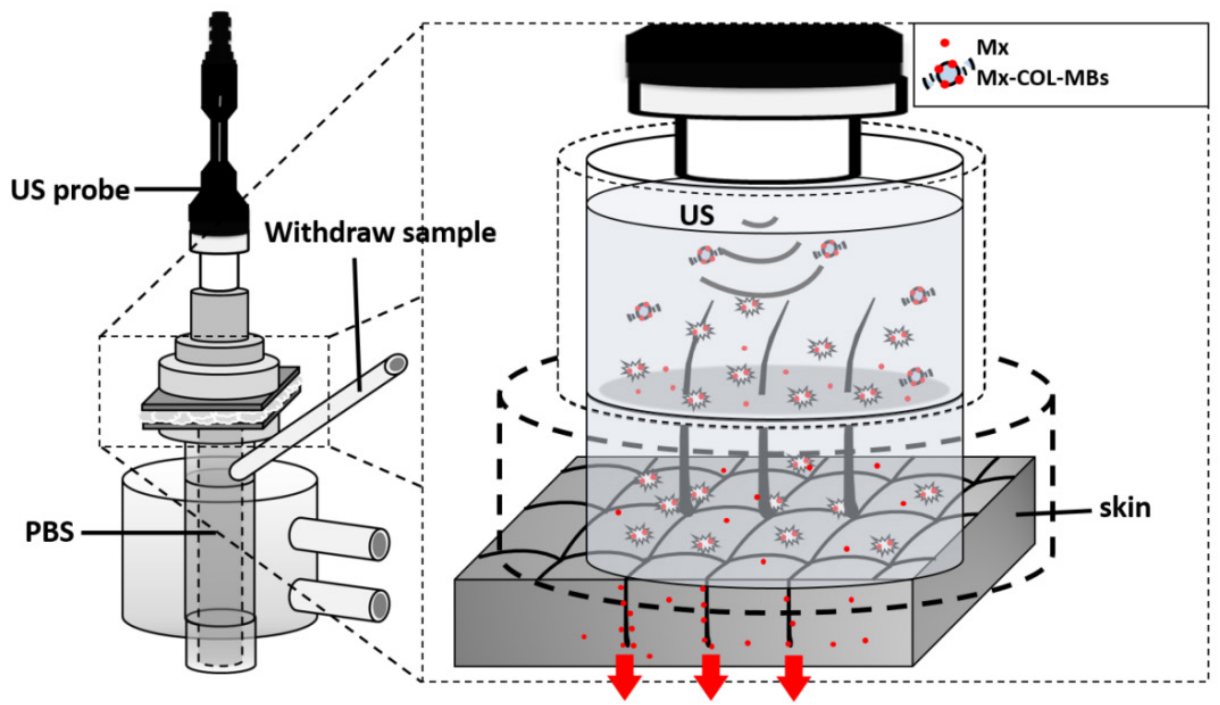

Figure 3: System setup for measuring in vitro skin penetration using static Franz diffusion cells. 


\section{Results}

\section{Preparation and characterization of Mx-COL-MBs}

The mean diameters of the MBs, COL-MBs, and Mx-COL-MBs were 1480, 4150, and 4500 nm (Fig. 4A). The zeta potentials of the MBs, COL-MBs and Mx-COL-MBs (with COL:Mx=1:1, 2:1 and 3:1) dispersed in an aqueous solution $(\mathrm{pH}=6.4$, resistance $=18.2 \mathrm{~m} \Omega$ ) were measured using a Nanoparticle analyzer (Horiba, Kyoto, Japan). Albumin is a negatively charged protein at $\mathrm{pH} 7$, and the MBs had a negative potential of $-8.52 \pm 0.81 \mathrm{mV}$ (mean $\pm \mathrm{SD})$. Upon $\mathrm{COL}$ and $\mathrm{Mx}$ conjugation, the surface potentials of the COL-MBs and Mx-COL-MBs reversed to $+20.23-25.23 \mathrm{mV}$ and $+0.41-21.01 \mathrm{mV}$ (Fig. $4 \mathrm{~B}$ and Table 1). The encapsulation efficiency of the Mx coated on the albumin MB shells was analyzed using an ELISA reader (Epoch, Biotek, Winooski, VT,
USA). The absorbance spectra of the human serum albumin (HSA) solution; Mx; MBs, Mx-COL, and Mx-COL-MBs after US sonication (destruction); and normal saline solution are shown in Fig. 5A. HSA and albumin-shelled MBs absorb light at $280 \mathrm{~nm}$, whereas Mx and Mx-COL-MBs absorb light at 230, 261, and $285 \mathrm{~nm}$. Figure 5B shows the calibration curves of $\mathrm{Mx}$ at various concentrations. The maximum loading efficiency of Mx on COL-MBs (with COL:Mx=1:1) was $14.87 \%(n=5)$. Figure $6 \mathrm{~A}-\mathrm{C}$ show scanning electron microscopy (SEM) images of the MBs, COL-MBs, and $\mathrm{Mx}-\mathrm{COL}-\mathrm{MBs}$, respectively. The composite structures of the albumin MB shell, COL-MB shell, and the Mx-COL-MB shell were observable by SEM, which indicated the presence of small protein particles on the MB surface that appeared to be smoother after COL coating and the inclusion of some nanoscale particles after Mx coating.

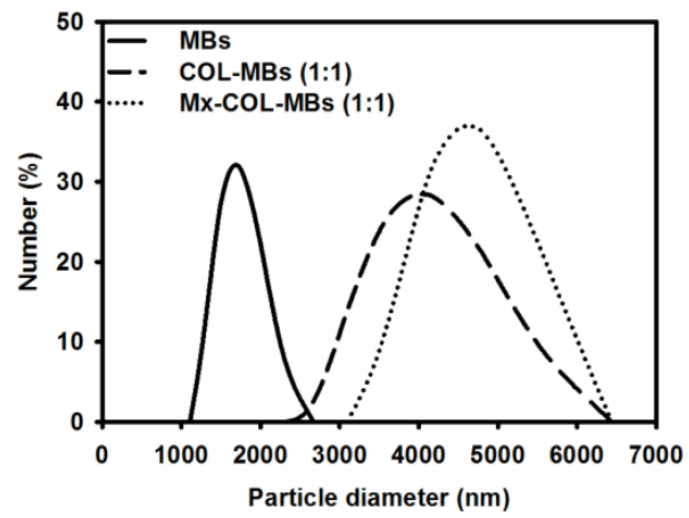

A

Figure 4: Quantification of the size distributions (A) and zeta potentials (B) of MBs, COL-MBs, and Mx-COL-MBs.
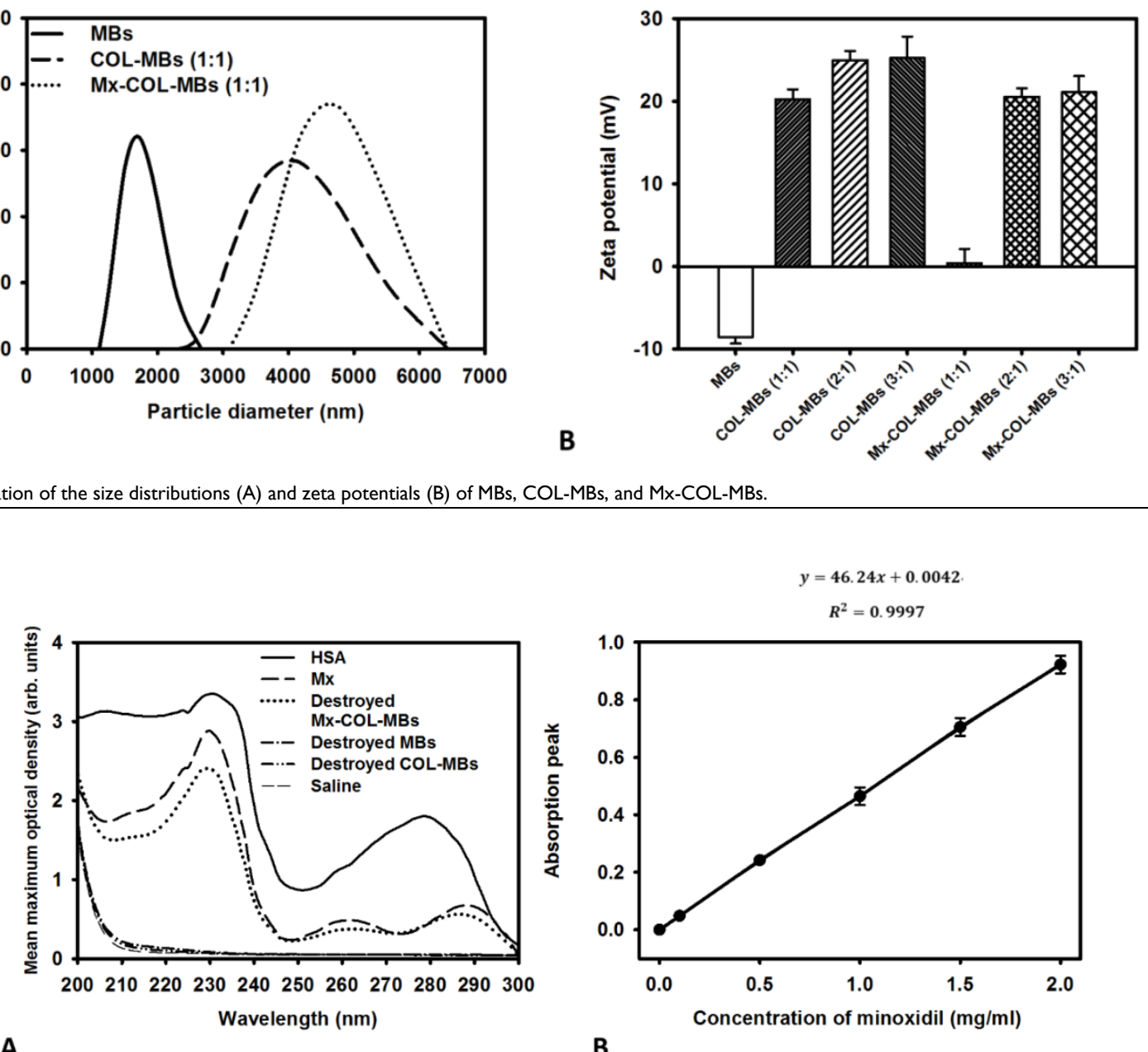

Figure 5: (A) Absorbance spectra of the HSA solution; Mx; Mx-COL-MBs, MBs, and COL-MBs after US sonication (destruction); and normal saline solution. (B) Calibration curve of the absorption of $M x$ versus its concentration. 


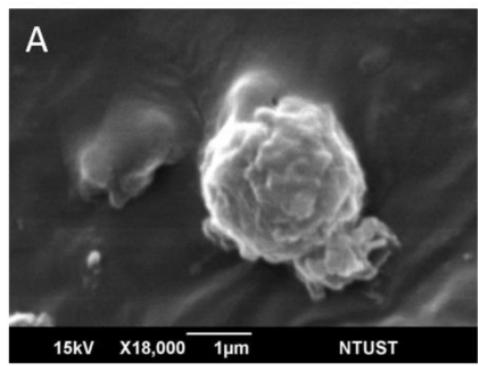

MBs

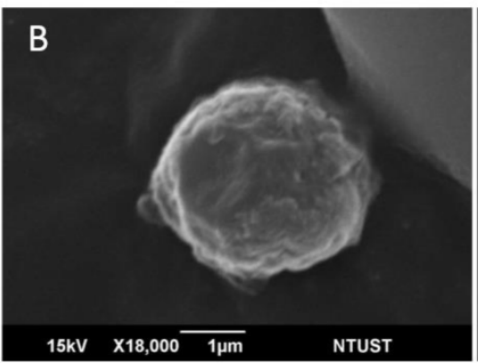

COL-MBs

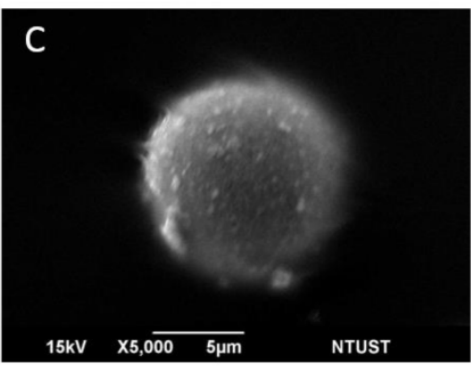

Mx-COL-MBs

Figure 6: Scanning electron microscopy (SEM) images of the (A) MBs, (B) COL-MBs, and (C) Mx-COL-MBs.

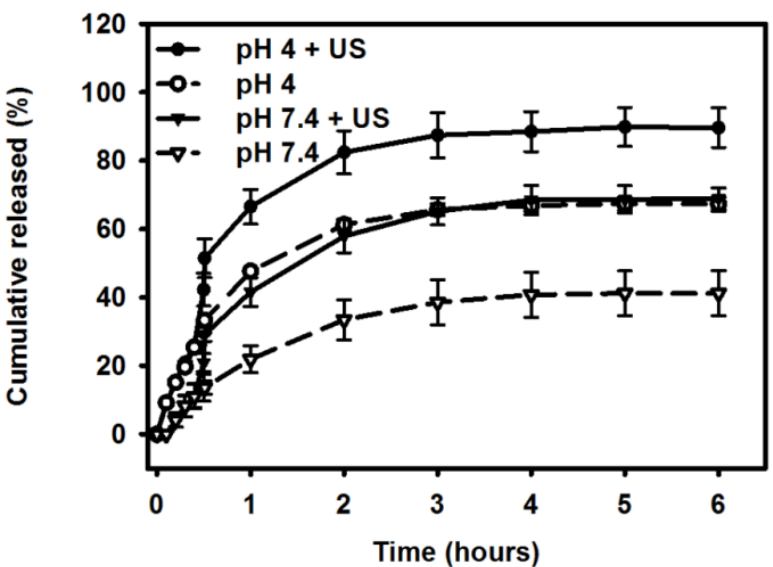

Figure 7: Comparative cumulative drug release of $M x$ after 6 hours from Mx-COL-MBs with and without US sonication in PBS ( $\mathrm{pH} 4$ and 7.4).

\section{In vitro release study}

Figure 7 shows the percentage cumulative drug release after 6 hours for Mx-COL-MBs and US combined with Mx-COL-MBs (US+Mx-COL-MBs) in phosphate-buffered saline (PBS; $\mathrm{pH} 4$ and 7.4). In the pH-7.4 environment, the diffusion (accumulative release percentage) of free $\mathrm{Mx}$ through the dialysis membrane relative to the control was only $13.6 \%$ over the first 0.5 hours, and this increased to $29.2 \%$ with US sonication. In the $\mathrm{pH}-4$ environment, the diffusion of free $\mathrm{Mx}$ through the dialysis membrane relative to the control was $30.3 \%$ over the first 0.5 hours, and this increased to $51.4 \%$ with US sonication. With US sonication, the in vitro release profile of the $\mathrm{Mx}$ showed a rapid release of just over $57 \%$ at $\mathrm{pH} 7.4$ and $80 \%$ at $\mathrm{pH} 4$ during the first 2 hours, and then a slower but sustained release of $\mathrm{Mx}$ from the Mx-COL-MBs to just over $68 \%$ at pH 7.4 and $89 \%$ at $\mathrm{pH} 4$ after 6 hours. Without US sonication, the amount of free drug suspension released across the dialysis membrane was reduced, to only $41.2 \%$ at $\mathrm{pH} 7.4$ and $67.3 \%$ at $\mathrm{pH} 4$ after 6 hours. These findings indicate that US energy can enhance drug release by $20-26 \%$ and also that the $\mathrm{pH}$ value affects the efficiency of $\mathrm{Mx}$ release from $\mathrm{Mx}-\mathrm{COL}-\mathrm{MBs}$.

\section{Measurements of penetration depth in pigskin}

In vitro skin permeation studies have demonstrated the ability of US-mediated MB cavitation to act on the hair follicle of drug as compared to US alone (Fig. 8). The intensities of the fluorescence signals transmitted from the model drug fluorescein isothiocyanate (FITC) detected in the histology sections were approximately 10.7 and 7.8 orders of magnitude higher in the US combined with MBs and penetrating FITC (US+MBs) group (Fig. 8F, penetration depth $=1856 \pm 45 \mu \mathrm{m})$ than in the FITC alone (Control) group (Fig. 8D, penetration depth $=312 \pm 19 \mu \mathrm{m})$ and the US combined with penetrating FITC (US) group (Fig. 8E, penetration depth $=405 \pm 23 \mu \mathrm{m})$, respectively, when the FITC solution was left for 6 hours. The detected fluorescence signal was weaker for the control and US-treated skin sites, and identical results were obtained for all hair follicles in all tissue samples for the US+MBs group.

\section{Control US US+MBs}

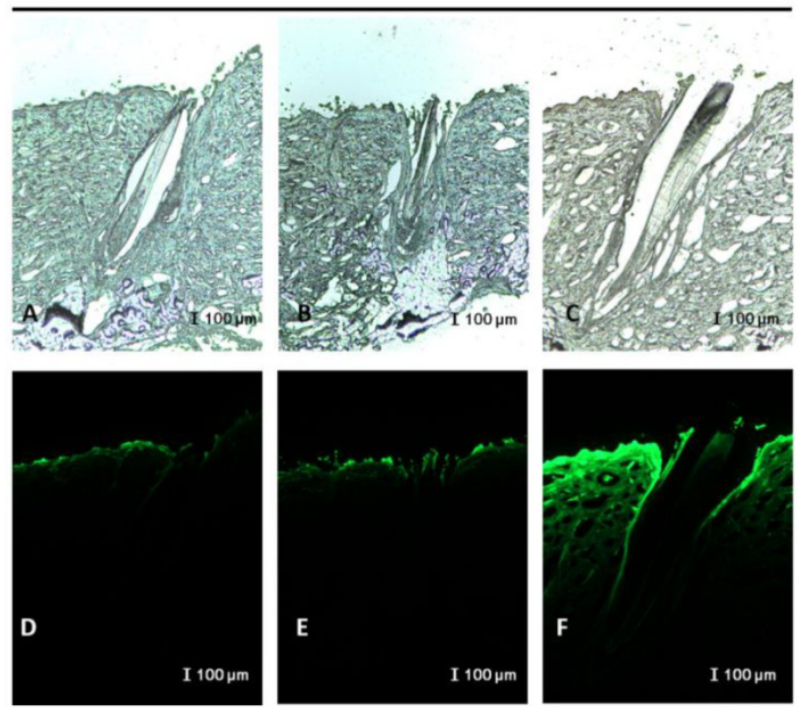

Figure 8: Bright-field microscopy (A-C) and fluorescence microscopy (D-F) images of the model drug FITC in the control, US, and US+MBs groups after the FITC solution had been left for 6 hours. 


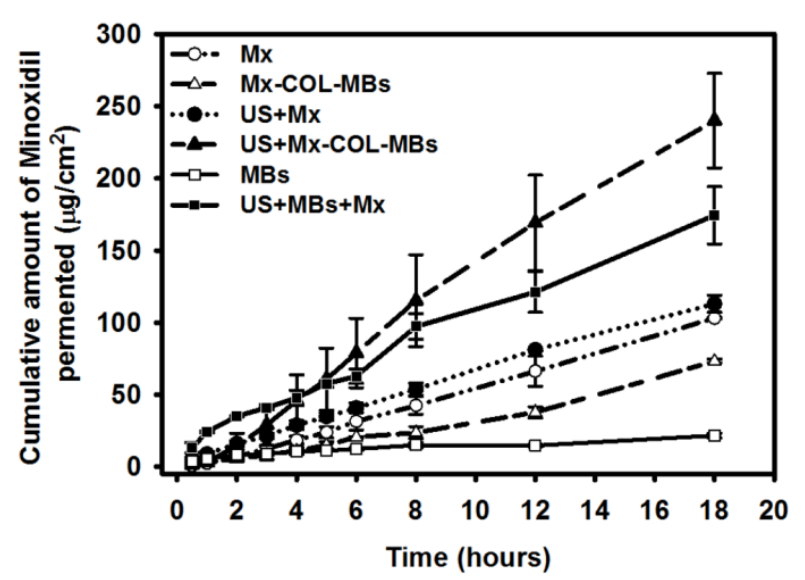

Figure 9: $M x$ concentrations in the MBs, Mx-COL-MBs, Mx, US+Mx, US+MBs+Mx, and US+Mx-COL-MBs groups for percutaneous penetration over 18 hours as analyzed using a UV-visual spectrophotometer. Data are mean and SD values.

\section{In vitro skin penetration by $\mathbf{M x}$}

Figure 9 shows the Mx concentrations in the six groups for percutaneous penetration over 18 hours as analyzed using a UV-visual spectrophotometer. The concentration in the US combined with MBs and penetrating $\mathrm{Mx}$ (US+MBs+Mx) group increased rapidly during the first 6 hours, reaching $62.9 \pm 5.0 \mu \mathrm{g} / \mathrm{ml}$, and then gradually leveled off between 8 to 18 hours. At 18 hours the concentration was significant higher $(p<0.05)$ in the US combined with Mx-COL-MBs (US+Mx-COL-MBs) group $(240.0 \pm 32.8 \mu \mathrm{g} / \mathrm{ml})$ than in the US+MBs+Mx $(174.3 \pm 19.8 \mu \mathrm{g} / \mathrm{ml})$, US combined with penetrating Mx (US+Mx) (113.0 $\pm 6.0 \mu \mathrm{g} / \mathrm{ml}), \mathrm{Mx}$ alone (Mx) $(103.1 \pm 0.5 \mu \mathrm{g} / \mathrm{ml}), \quad$ Mx-COL-MBs alone (Mx-COL-MBs) $(73.3 \pm 1.4 \mu \mathrm{g} / \mathrm{ml})$, and MBs alone (MBs) $(21.7 \pm 1.6 \mu \mathrm{g} / \mathrm{ml})$ groups. The concentration differed significantly $(p<0.05)$ between the MBs, $\mathrm{Mx}-\mathrm{COL}-\mathrm{MBs}, \mathrm{Mx}$ or US+Mx, US+MBs+Mx, and US+Mx-COL-MBs groups. Combining US with Mx-COL-MBs and MBs+Mx resulted in 2.3 and 1.7 times the penetration and deposition, respectively, of $\mathrm{Mx}$ relative to the $\mathrm{Mx}$ group. Table 2 indicates that the amounts of Mx that were deposited in the skin and appeared to be higher in the Mx group than in the US $+M x$ groups $(p<0.01)$, and significantly so compared to the Mx-COL-MBs, US+MBs+Mx and US+Mx-COL-MBs groups $(p<0.001)$.

\section{Animal treatments}

Figure 10A shows photographs of mouse skin in a completely untreated animal (day 1) and in the C, $\mathrm{Mx}$, US+Mx, US+MBs+Mx, and US+Mx-COL-MBs groups at various time points after treatment. At day 10, the skin brightness for the five mice in the US+Mx-COL-MBs group was more effectively decreased compared to the US+MBs+Mx (decreased in three mice), US+Mx (decreased in one mouse), $\mathrm{Mx}$ (decreased in one mouse), and C (not decreased in any mice) groups. At day 14, the hair growth was greater in all mice in the US+Mx-COL-MBs group than in the other four groups. Figure 10B demonstrates the effects of $\mathrm{Mx}$ on dorsal hair growth over 21 days. At days 10 and 14 , the growth rates in the US+Mx-COL-MBs group had increased by $22.6 \%$ and $64.7 \%$, respectively. At that time point there were obvious significant differences $(p<0.05)$ between the US+Mx-COL-MBs group and the other four groups. At day 16, the growth rate had reached a plateau in the US+Mx-COL-MBs group, with an increase of $84.2 \%$, while the growth rates in the C, Mx, US+Mx, and US+MBs+Mx groups had increased by $49.2 \%$, $45.5 \%, 64.5 \%$, and $83.5 \%$, respectively. At that time point the growth rate did not differ significantly between the US+Mx-COL-MBs and US+MBs+Mx groups $(p>0.05)$.

The histology images in Fig. 11 indicate that no skin damage was evident in any of the US treatment groups. Histological analysis of transverse sections (Fig. 11A-E) revealed that there were significant increases in skin thickness after 21 days of $\mathrm{Mx}$ application, in hair length after 21 days of US application, and in hair length in the US+MBs+Mx and US+Mx-COL-MBs groups. The enhancements of skin thickness and hair length were greatest in the US+Mx-COL-MBs group. Moreover, the combination of US and MBs promoted the elongation of hair follicles from the epidermis down to the subcutis in vertical sections. The histological analysis of coronal sections (Fig. 11F-J) revealed that the number of hair follicles was not increased after treatment $(P>0.05)$. The diameter of the keratinized hair shaft and the hair follicle size were both increased in the US+Mx, US+MBs+Mx, and US+Mx-COL-MBs groups (especially the latter two).

Table 2. Permeated amount of $M x$ at 18 hours, deposited on the skin and penetrated across the skin. Data are mean $\pm S D$ values. Mx, penetrating Mx alone; US, US combined with penetrating Mx; US+MBs, US combined with MBs and penetrating Mx; US+Mx-COL-MBs, US combined with Mx-COL-MBs.

\begin{tabular}{llll}
\hline Group & Skin weight $(\mathrm{g})$ & $\begin{array}{l}\text { Amount of Mx } \\
\text { deposited on skin }(\mu \mathrm{g} / \mathrm{ml})\end{array}$ & $\begin{array}{l}\text { Amount of Mx } \\
\text { penetrated across skin }(\mu \mathrm{g} / \mathrm{ml})\end{array}$ \\
\hline Mx & $0.1659 \pm 0.0325$ & $52.42 \pm 9.55$ & $103.10 \pm 0.51$ \\
US+Mx & $0.1557 \pm 0.0198$ & $33.02 \pm 1.56$ & $113.01 \pm 5.95$ \\
permeated $(\mu \mathrm{g} / \mathrm{ml})$
\end{tabular}


A

Day 7

C

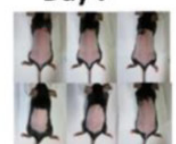

$4 \times$

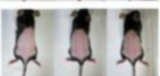

$\mathrm{Mx}$

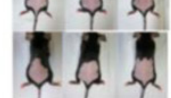

T)

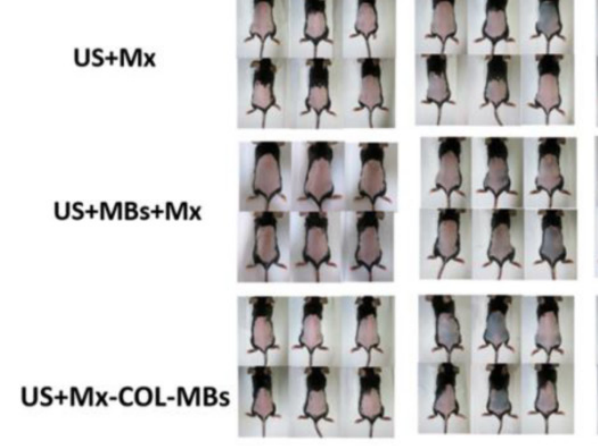

US+Mx

B

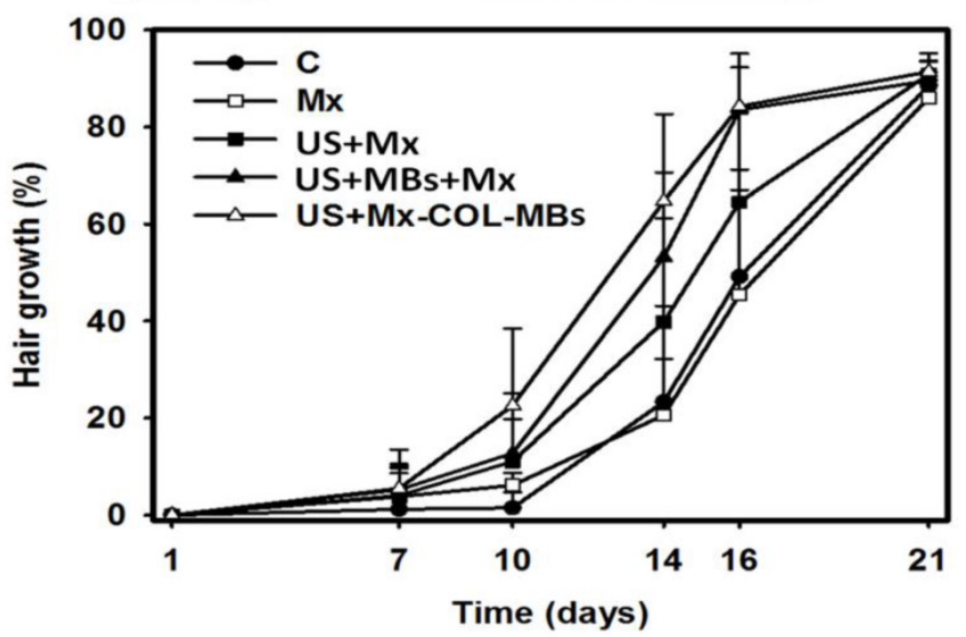

Day 10
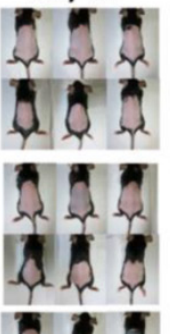

Day 14
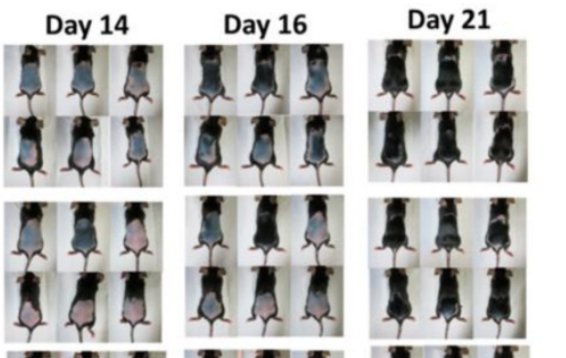

(11
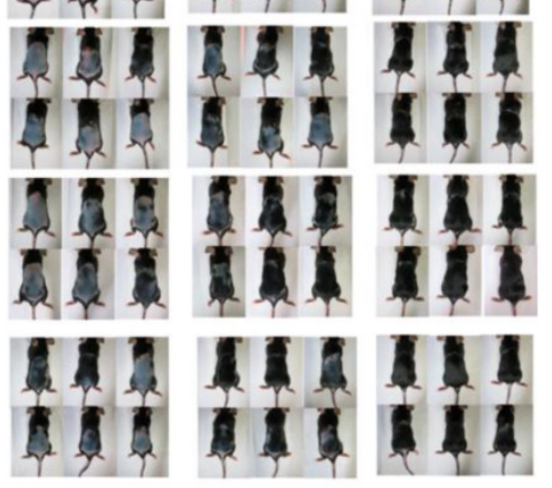

Figure 10: (A) Gross observations of the dorsal skin of C57BL6 mice. The dorsal skin surfaces of the mice were shaved, and then test compounds were topically applied for 3 weeks. C, no treatment; Mx, penetrating Mx alone; US, US combined with penetrating Mx; US+MBs, US combined with MBs and penetrating Mx; US+Mx-COL-MBs, US combined with Mx-COL-MBs. (B) Quantification of hair growth rates on the dorsal skin after shaving the hair in various mouse groups over 21 days. Data are mean and SD values.

\section{C}

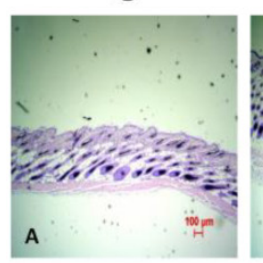

$\mathrm{Mx}$
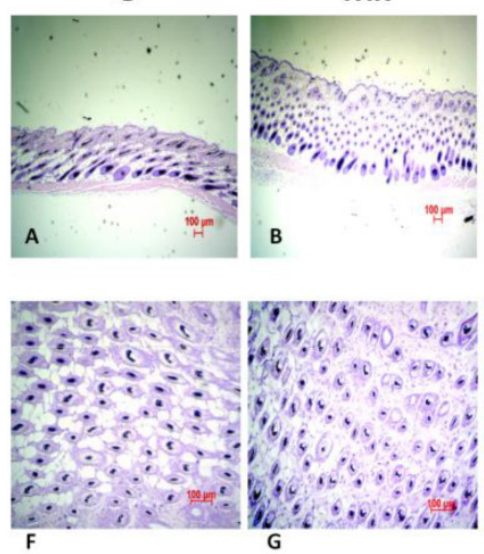

\section{.}

\section{.}




\section{Discussion}

Serum albumin has been widely used in nanoscale- and microscale-particle drug carrier systems due to its nontoxic, nonantigenic, and biodegradable properties [28]. Modifying the anionic side-chain carboxylic groups of albumin with a cationic amino group produces cationic serum albumin, which has low cytotoxicity and good biocompatibility since the cationization does not destroy the protein structure and its properties [29]. In the present study, a biodegradable, low-molecular-weight cationic polymer (COL) was added to the albumin-shelled MBs to form stable cationic COL-MBs. A previous study showed that chitosan microparticles containing different proportions of $\mathrm{Mx}$ and polymer had a high encapsulation efficiency (82\%) of Mx [30]. That study found that larger proportions of $\mathrm{Mx}$ in relation to the polymer resulted in a higher percentage of $\mathrm{Mx}$ encapsulation. In our study (see Table 1), Mx-COL-MBs with COL:Mx=1:1 had the highest loading efficiency of $\mathrm{Mx}$ on COL-MBs, and this did not vary in proportion with the ratios of MX and COL, which may be due to limitations of the drug loading capacity of the MBs owing to the limited surface area [31]. The zeta potential in 1:1, 2:1 or 3:1 COL-MBs are very similar since the limited surface area of MBs. Moreover, in some 2:1 or 3:1 COL-MBs, the extra free COL escaped from MBs may be absorbed with $\mathrm{Mx}$ and decreased the loading efficiency.

The in vitro investigation found that while the application of US energy can enhance drug release, using a low $\mathrm{pH}$ value can also increase the efficiency of Mx release from Mx-COL-MBs. Since chitosan is a $\mathrm{pH}$-sensitive polymer, the $\mathrm{pH}$ and the nature of the dispersive medium affect the swelling properties of chitosan. The swelling properties of COL in low $\mathrm{pH}$ may lead to unstable conjugation between $\mathrm{Mx}$ and COL-MBs, and increase the $\mathrm{Mx}$ released from COL-MBs. This property can be utilized to improve the $\mathrm{Mx}$ delivery into the scalp since the $\mathrm{pH}$ of the scalp (like the rest of the skin) is around 5.5 [32]. In experiments of the FITC penetration depth in pigskin, combining US with MBs resulted in more efficient and deeper penetration into the hair follicles compared to using US alone, and hence it can therefore deliver a drug closer to the target structure of interest in the hair follicles.

The in vitro permeation profiles of $\mathrm{Mx}$ through the skins are shown in Fig. 9 and Table 2, which indicate that the drug concentration in the receptor chambers increased steadily over time. Although the concentrations in the US+MBs+Mx group increased more rapidly relative to the other groups during the first 6 hours, it then became closer to or even lower than the US+Mx-COL-MBs group from 6 to 18 hours. Our results indicate that combining US with Mx-COL-MBs increased the total delivered concentration of $\mathrm{Mx}$ and did not influence the time trend of Mx delivery relative to using US alone. Combining US and MBs can initially rapidly improve the permeation of free Mx molecules, with this effect gradually leveling off between 8 and 18 hours.

C57BL/ 6 mice are commonly used to screen agents for promoting hair growth, because their truncal pigmentation is dependent on their follicular melanocytes, which produce pigment only during the anagen phase [33]. In our study, the untreated animals also showed considerable hair growth on the 21th day after the treatment. The proportion of mice that produced pigment on the $10^{\text {th }}$ day was highest in the US+Mx-COL-MBs group and lowest in the US+Mx and US+MBs+Mx groups. This was due to $\mathrm{Mx}$ shortening the telogen phase while having no effect on the duration of the anagen phase [34]. US combined with MBs and US combined with Mx-COL-MBs increased the effects of $\mathrm{Mx}$ so as to shorten the telogen phase. Similar to some previous studies, our histological study indicated that $\mathrm{Mx}$ induced thick and long hair after 21 days of topical application, and it also promoted the elongation of hair follicles from the epidermis down to the subcutis in vertical sections (Fig. 11A-E) [35]. The application of US+Mx, US+MBs+Mx and US+Mx-COL-MBs enhanced these effects. In these three groups the number of hair follicles was not increased, but the diameter of the hair shafts and the size of the hair follicles were both increased more significantly than in the Mx group, especially in the US+MBs+Mx and US+Mx-COL-MBs groups.

\section{Conclusion}

This study provides a new integrated transdermal drug delivery platform for enhancing and monitoring the delivery of $\mathrm{Mx}$ to hair follicles by utilizing multifunctional MBs. US can locally treat the skin, and the application of the new Mx-COL-MBs significantly improves the deposition of $\mathrm{Mx}$. The combined use of US and Mx-COL-MBs provides an important means to increase the rate of hair growth and the diameter of keratinized hair shafts. Most importantly, US combined with Mx-COL-MBs can significantly enhance hair growth in water without requiring the use of any chemical enhancement method to increase the skin permeability.

\section{Acknowledgments}

This work was supported in part by grants from the Ministry of Science and Technology, Taiwan 
(MOST103-2221-E-011-004-MY3 to A.-H.L. and MOST104-2314-B-016-032-MY3 to C.-H.W.), the Tri-Service General Hospital (TSGH-NTUST-105-01 to A.-H.L. and TSGH-C105-008 to C.-H.W.), and National Defense Medical Research grants (MAB104-056 to H.-K.S. and MAB105-016 to C.-H.W.).

\section{Competing Interests}

The authors have declared that no competing interest exists.

\section{References}

1. Springer K, Brown M, Stulberg DL. Common hair loss disorders. Am Fam Physician. 2003; 68(1): 93-102.

2. Rathnayake D, Sinclair R. Male androgenetic alopecia. Expert Opin Pharmacother. 2010; 11(8): 1295-304.

3. Price VH. Androgenetic alopecia in women. J Investig Dermatol Symp Proc. 2003; 8(1): 24-7.

4. Liao AH, Chou HY, Hsieh YL, et al. Enhanced Therapeutic Epidermal Growth Factor Receptor (EGFR) Antibody Delivery via Pulsed Ultrasound with Targeting Microbubbles for Glioma Treatment. J Med Biol Eng. 2015; 35(2): 156-64.

5. Limas CJ, Freis ED. Minoxidil in severe hypertension with renal failure. Effect of its addition to conventional antihypertensive drugs. Am J Cardiol. 1973; 31(3): 355-61.

6. Mehta PK, Mamdani B, Shansky RM, et al. Severe hypertension. Treatment with minoxidil. JAMA. 1975; 233(3): 249-52.

7. Zappacosta AR. Reversal of baldness in patient receiving minoxidil for hypertension. N Engl J Med. 1980; 303(25): 1480-81.

8. Messenger AG, Rundegren J. Minoxidil: mechanisms of action on hair growth. Br J Dermatol. 2004; 150(2): 186-94.

9. Gupta AK, Foley KA. 5\% Minoxidil: treatment for female pattern hair loss. Skin Therapy Lett. 2014; 19(6): 5-7.

10. Olsen EA, Dunlap FE, Funicella $\mathrm{T}$, et al. A randomized clinical trial of $5 \%$ topical minoxidil versus $2 \%$ topical minoxidil and placebo in the treatment of androgenetic alopecia in men. J Am Acad Dermatol. 2002; 47(3): 377-85.

11. Olsen EA, Whiting D, Bergfeld $W$, et al. A multicenter, randomized, placebocontrolled, double-blind clinical trial of a novel formulation of $5 \%$ minoxidil topical foam versus placebo in the treatment of androgenetic alopecia in men. J Am Acad Dermatol. 2007; 57(5): 767-74.

12. Tata S, Flynn GL, Weiner ND. Penetration of minoxidil from ethanol/propylene glycol solutions: effect of application volume and occlusion. J Pharm Sci. 1995; 84(6): 688-91.

13. Friedman ES, Friedman PM, Cohen DE, et al. Allergic contact dermatitis to topical minoxidil solution: etiology and treatment. J Am Acad Dermatol. 2002; 46(2): 309-13.

14. Gelfuso GM, Gratieri T, Simão PS, et al. Chitosan microparticles for sustaining the topical delivery of minoxidil sulphate. J Microencapsul. 2011; 28(7): 650-8.

15. Jeong K, Lee YJ, Kim JE, et al. Repeated microneedle stimulation induce the enhanced expression of hair-growth-related genes. Int J Trichology. 2012; 4(2): $117-30$.

16. Chandrashekar BS, Yepuri V, Mysore V. Alopecia areata-successful outcome with microneedling and triamcinolone acetonide. J Cutan Aesthet Surg. 2014; 7(1): 63-64.

17. Wu D, Quan YS, Kamiyama F, et al. Improvement of transdermal delivery of sumatriptan succinate using a novel self-dissolving microneedle array fabricated from sodium hyaluronate in rats. Biol Pharm Bull. 2015; 38(3): 365-73.

18. Park D, Yoon J, Park J, et al. Transdermal drug delivery aided by an ultrasound contrast agent: an in vitro experimental study. Open Biomed Eng J. 2010; 4: $56-62$.

19. Park D, Ryu H, Kim HS, et al. Sonophoresis using ultrasound contrast agents for transdermal drug delivery: an in vivo experimental study. Ultrasound Med Biol. 2012; 38(4): 642-50.

20. Liao $\mathrm{AH}$, et al. Penetration depth, concentration and efficiency of transdermal a-arbutin delivery after ultrasound treatment with albumin-shelled microbubbles in mice. Drug Deliv; in press.

21. Liao $\mathrm{AH}, \mathrm{Ho} \mathrm{HC}$, Lin $\mathrm{YC}$, et al. Effects of microbubble size on ultrasound-induced transdermal delivery of high-molecular-weight drugs. PLoS One. 2015; 10(9): e0138500.

22. Ahn BN, Kim JA, Himaya SW, et al. Chitooligosaccharides attenuate UVB-induced damages in human dermal fibroblasts. Naunyn Schmiedebergs Arch Pharmacol. 2012; 385(1): 95-102.

23. Liao $\mathrm{AH}$, Hsieh $\mathrm{YL}$, $\mathrm{Ho} \mathrm{HC}$ et al. Effects of microbubble size on ultrasound-mediated gene transfection in auditory cells. Biomed Res Int. 2014; 840852 .

24. Shih $\mathrm{CP}$, Chen $\mathrm{HC}$, Chen $\mathrm{HK}$, et al. Ultrasound-aided microbubbles facilitate the delivery of drugs to the inner ear via the round window membrane. J Control Release. 2013; 167(2): 167-74.
25. Gao J, Wang $\mathrm{Z}$, Liu $\mathrm{H}$, et al. Liposome encapsulated of temozolomide for the treatment of glioma tumor: preparation, characterization and evaluation. Drug Discov Ther. 2015; 9(3): 205-12.

26. Mak WC, Patzelt A, Richter $\mathrm{H}$, et al. Triggering of drug release of particles in hair follicles. J Control Release. 2012; 160(3): 509-14

27. Tsai $\mathrm{YH}$, Lee KF, Huang $\mathrm{YB}$, et al. In vitro permeation and in vivo whitening effect of topical hesperetin microemulsion delivery system. Int J Pharm. 2010; 388(1-2): 257-62.

28. Rhaese S, von Briesen H, Rübsamen-Waigmann $H$, et al. Human serum albumin-polyethylenimine nanoparticles for gene delivery. J Control Release. 2003; 92(1-2): 199-208.

29. Fischer D, Bieber T, Brüsselbach S, et al. Cationized human serum albumin as a non-viral vector system for gene delivery? Characterization of complex formation with plasmid DNA and transfection efficiency. Int J Pharm. 2001; 225(1-2): 97-111.

30. Gelfuso GM, Gratieri T, Simão PS, et al. Chitosan microparticles for sustaining the topical delivery of minoxidil sulphate. J Microencapsul. 2011; 28(7): 650-8.

31. Sirsi SR, Fung C, Garg $S$, et al. Lung surfactant microbubbles increase lipophilic drug payload for ultrasound-targeted delivery. Theranostics. 2013; 3(6): 409-19.

32. Gavazzoni Dias MF, de Almeida AM, Cecato PM, et al. The shampoo $\mathrm{pH}$ can affect the hair: myth or reality? Int J Trichology. 2014; 6(3): 95-9.

33. Plonka PM, Michalczyk D, Popik M, et al. Splenic eumelanin differs from hair eumelanin in C57BL/ 6 mice. Acta Biochim Pol. 2005; 52(2): 433-41.

34. 34. Mori $\mathrm{O}$, Uno $\mathrm{H}$. The effect of topical minoxidil on hair follicular cycles of rats. J Dermatol. 1990; 17(5): 276-81.

35. Oh JY, Park MA, Kim YC. Peppermint oil promotes hair growth without toxic signs. Toxicol Res. 2014; 30(4): 297-304. 